\title{
Comparison of three Frisch methods for errors-in-variables identification
}

\author{
Mei Hong*, Torsten Söderström*, Umberto Soverini** \\ and Roberto Diversi** \\ * Division of Systems and Control, \\ Department of Information Technology, Uppsala University, \\ P O Box 337, SE-75105 Uppsala, Sweden \\ ** Dipartimento di Elettronica, \\ Informatica e Sistemistica, University of Bologna, \\ Viale del Risorgimento 2, 40136 Bologna, Italy
}

August 29, 2007

\begin{abstract}
The errors-in-variables framework concerns static or dynamic systems whose input and output variables are affected by additive noise. Several estimation methods have been proposed for identifying dynamic errors-invariables models. One of the more promising approaches is the so-called Frisch scheme. This report decribes three different estimation criteria within the Frisch context and compares their estimation accuracy on the basis of the asymptotic covariance matrices of the estimates. Some final numerical examples support the theoretical results and analyze the behaviour of the methods in case of finite number of data.
\end{abstract}

\section{Introduction}

The Errors-In-Variables (EIV) framework concerns static or dynamic systems whose input and output variables are affected by additive noise. These models play an important role in several engineering applications like, for example, time 
series modeling, direction-of-arrival estimation, blind channel equalization, and many other signal and image processing problems; see [17, 18].

One of the first solutions for the identification of dynamic EIV models was given in [11] and [1]. After these pioneer works, this topic has been reproposed by several authors with different approaches. An overview of EIV identification methods can be found in [13].

Among these methods, the so-called Frisch scheme is one of the more interesting. Its roots are in [8], with reference to static problems; the extension to dynamic systems was proposed in [3].

One of the main difficulties of the method concerns its application to real cases, when for several reasons (limited number of the available samples, nonlinearities in the system, etc.) the assumptions behind the scheme are not exactly satisfied. In these cases the identification procedure does no more lead to a single solution, unless a selection criterion is introduced.

One of the first criteria that has shown remarkable robustness properties was originally proposed in [2] and further in [5]. This criterion, denoted as Frisch$\mathrm{SR}$, relies on the shift properties of time-invariant systems and is based on rank deficiency conditions of the noise-free covariance matrix. A recent analysis [9] has shown that this method is equivalent to the Bias-Eliminating Least Squares (BELS) procedure proposed in [19] under the same user's choices.

Another robust criterion, characterized by a high level of estimation accuracy, was proposed in [4]. This approach, denoted as Frisch-CM, relies on a comparison between the true and estimated statistical properties of the EIV system residuals. A theoretical analysis concerning the accuracy of the estimates obtained with this method was given in [12].

A third criterion, characterized by a good compromise between computational efficiency and estimation accuracy, was introduced in [6]. This method, denoted as Frisch-YW, relies on the properties of the high order Yule-Walker equations and is characterized by the same asymptotic properties of the Frisch-SR, as shown in Section 3.

For the three Frisch alternatives, it is of interest to know which one gives the best estimation accuracy. This report compares these criteria on the basis of the asymptotic covariance matrices of the estimates.

It will be shown that for moderate signal-to-noise ratios (SNR) no alternative is better than the other, both for the system parameters and noise variances. On the contrary, for high SNR the noise variances can, in general, be better estimated when the Frisch-CM criterion is used.

The report is organized as follows. Section 2 describes the EIV identification problem and analyses its solution by means of the Frisch scheme. In particular the three different Frisch scheme criteria are briefly recalled. Some algorithmic aspects of these variants are then discussed in Section 3. Section 4 contains the 
numerical comparison and theoretic analysis of the asymptotic covariance matrices of the estimates obtained with the three different Frisch methods. Section 5 compares the proposed methods in presence of finite data by means of Monte Carlo simulations. Finally, some concluding remarks are reported is Section 5.

\section{Problem statement and notations}

Consider a linear and single input single output (SISO) system given by

$$
A\left(q^{-1}\right) y_{0}(t)=B\left(q^{-1}\right) u_{0}(t)
$$

where $u_{0}(t)$ and $y_{0}(t)$ are the noise-free input and output, respectively. Further, $A\left(q^{-1}\right)$ and $B\left(q^{-1}\right)$ are polynomials described as

$$
\begin{aligned}
& A\left(q^{-1}\right)=1+a_{1} q^{-1}+\cdots+a_{n_{a}} q^{-n_{a}} \\
& B\left(q^{-1}\right)=b_{0}+b_{1} q^{-1}+\cdots+b_{n_{b}} q^{-n_{b}}
\end{aligned}
$$

We assume that the observations are corrupted by additive measurement noises $\tilde{u}(t)$ and $\tilde{y}(t)$. The available signals are in discrete time and of the form

$$
u(t)=u_{0}(t)+\tilde{u}(t), \quad y(t)=y_{0}(t)+\tilde{y}(t) .
$$

The following assumptions are introduced.

A1. The dynamic system (1) is asymptotically stable, observable and controllable.

A2. The polynomial degrees $n_{a}$ and $n_{b}$ are a priori known.

A3. The true input $u_{0}(t)$ is a zero-mean stationary ergodic random signal, that is persistently exciting of sufficiently high order.

A4. The input noise $\tilde{u}(t)$ and the output noise $\tilde{y}(t)$ are both independent of $u_{0}(t)$ and mutually independent white Gaussian noise sequences of zero mean, and variances $\lambda_{u}$ and $\lambda_{y}$, respectively.

The problem of identifying this EIV system is concerned with consistently estimating the parameter vector $\theta_{0}=\left(a_{1} \ldots a_{n_{a}} b_{0} \ldots b_{n_{b}}\right)^{T}$ and the noise variances $\lambda_{u}$ and $\lambda_{y}$ from the measured noisy data $\{u(t), y(t)\}_{t=1}^{N}$.

We introduce the regressor vector

$$
\begin{aligned}
& \varphi(t)=\left(-y(t-1) \cdots-y\left(t-n_{a}\right) \quad u(t) \ldots u\left(t-n_{b}\right)\right)^{T} \\
& =\left(-y_{0}(t-1) \cdots-y_{0}\left(t-n_{a}\right) \quad u_{0}(t) \ldots u_{0}\left(t-n_{b}\right)\right)^{T} \\
& +\left(-\tilde{y}(t-1) \cdots-\tilde{y}\left(t-n_{a}\right) \quad \tilde{u}(t) \ldots \tilde{u}\left(t-n_{b}\right)\right)^{T} \\
& \triangleq \varphi_{0}(t)+\tilde{\varphi}(t)
\end{aligned}
$$


where $\varphi_{0}(t)$ and $\tilde{\varphi}(t)$ denote the noise-free part and the noise contribution part of $\varphi(t)$, respectively.

For convenience, we utilize the extended regressor $\phi(t)$ and the true extended parameter vector $\Theta_{0}$ as

$$
\phi(t)=\left(\begin{array}{ll}
-y(t) & \varphi^{T}(t)
\end{array}\right)^{T}, \quad \Theta_{0}=\left(\begin{array}{ll}
1 & \theta_{0}^{T}
\end{array}\right)^{T} .
$$

In similar way the extended vectors $\phi_{0}(t)$ and $\tilde{\phi}(t)$ can be defined.

Some further expressions are introduced for the regressor vector and the system parameter vector, partitioned as

$$
\begin{aligned}
& \varphi_{u}(t)=\left(\begin{array}{c}
u(t) \\
\vdots \\
u\left(t-n_{b}\right)
\end{array}\right), \quad \varphi_{y}(t)=\left(\begin{array}{c}
-y(t-1) \\
\vdots \\
-y\left(t-n_{a}\right)
\end{array}\right), \quad \phi_{y}(t)=\left(\begin{array}{c}
-y(t) \\
\varphi_{y}(t)
\end{array}\right) \\
& \theta=\left(\begin{array}{c}
\mathbf{a} \\
\mathbf{b}
\end{array}\right), \quad \mathbf{a}=\left(\begin{array}{c}
a_{1} \\
\vdots \\
a_{n_{a}}
\end{array}\right), \quad \mathbf{b}=\left(\begin{array}{c}
b_{1} \\
\vdots \\
b_{n_{b}}
\end{array}\right), \quad \overline{\mathbf{a}}=\left(\begin{array}{c}
1 \\
\mathbf{a}
\end{array}\right)
\end{aligned}
$$

For a general random process $x(t)$, we define its covariance function $r_{x}(\tau)$ as:

$$
r_{x}(\tau)=E(x(\tau) x(t-\tau)), \quad \tau=0, \pm 1, \pm 2, \ldots
$$

Further, the cross-covariance matrix between two random vectors $x(t)$ and $y(t)$ and the cross-covariance vector between random vector $x(t)$ and random variable $z(t)$ are denoted as

$$
R_{x y}=E x(t) y^{T}(t), \quad r_{x z}=E x(t) z(t) .
$$

\subsection{Three variants of the Frisch scheme}

The Frisch scheme was first proposed by Ragnar Frisch, [8] in 1934. It was developed to identify dynamic EIV systems in [3] and was further elaborated in $[2,4,5,6]$. Consider the relation

$$
\phi_{0}^{T}(t) \Theta_{0}=-A_{0}\left(q^{-1}\right) y_{0}(t)+B_{0}\left(q^{-1}\right) u_{0}(t)=0 .
$$

It follows from (9) that

$$
R_{\phi_{0} \phi_{0}} \Theta_{0}=E\left(\phi_{0}(t) \phi_{0}(t)^{T}\right) \Theta_{0}=\mathbf{0} .
$$


Hence matrix $R_{\phi_{0} \phi_{0}}$ is singular (positive semidefinite), with at least one eigenvalue equal to zero. Since it holds that $R_{\phi \phi}=R_{\phi_{0} \phi_{0}}+R_{\tilde{\phi} \tilde{\phi}}$, relation (10) can also be expressed as

$$
\left(R_{\phi \phi}-R_{\tilde{\phi} \tilde{\phi}}\right) \Theta_{0}=\mathbf{0}
$$

where

$$
R_{\tilde{\phi} \tilde{\phi}}=\left(\begin{array}{cc}
\lambda_{y} I_{n_{a}+1} & \mathbf{0} \\
\mathbf{0} & \lambda_{u} I_{n_{b}+1}
\end{array}\right)
$$

The relations (11) and (12) are the basis for the Frisch method. They constitute a system of $2 n+1$ equations in $2 n+2$ unknowns. The solution of this system of equations can thus be expressed, in general, as a function of one variable, for example $\lambda_{u}$. In fact, if an estimate of $\lambda_{u}$ is available, the output noise variance $\lambda_{y}$ that satisfies (11) is given by

$$
\lambda_{y}=\lambda_{\min }\left(R_{\phi_{y} \phi_{y}}-R_{\phi_{y} \varphi_{u}}\left(R_{\varphi_{u} \varphi_{u}}-\lambda_{u} I_{n_{b}+1}\right)^{-1} R_{\varphi_{u} \phi_{y}}\right),
$$

where $\lambda_{\min }(R)$ denotes the smallest eigenvalue of $R$ [3].

Therefore, it can be stated that, in general, the solution of the nonlinear system of equations (11) and (12) is univocally determined if at least one additional equation is introduced. In this paper three alternatives will be considered.

- One choice is to evaluate the Frisch equations for an extended model by using an additional extended regressor vector $\underline{\varphi}(t)$. This method was proposed in $[2,5]$.

- The second alternative is to compute residuals and compare their statistical properties with what can be predicted from the model, proposed in [4].

- The third alternative is to use the Yule-Walker equations, proposed recently in [6].

For the first alternative the extended Frisch equation will be

$$
\left(R_{\bar{\phi} \bar{\phi}}-R_{\overline{\tilde{\phi}} \tilde{\tilde{\phi}}}\right) \bar{\Theta}_{0}=\mathbf{0},
$$

where

$$
\bar{\phi}=\left(\begin{array}{c}
\phi(t) \\
\underline{\varphi}(t)
\end{array}\right), \quad \overline{\tilde{\phi}}=\left(\begin{array}{c}
\tilde{\phi}(t) \\
\tilde{\varphi}(t)
\end{array}\right), \quad \bar{\Theta}_{0}=\left(\begin{array}{c}
\Theta_{0} \\
\mathbf{0}
\end{array}\right) .
$$

The model extension can, for example, mean that an additional $A$ parameter is appended. In that case, $\underline{\varphi}(t)=-y\left(t-n_{a}-1\right)$. Another possibility is to append 
an additional $B$ parameter, leading to $\varphi(t)=u\left(t-n_{b}-1\right)$. Furthermore, it is also possible to let $\varphi(t)$ be a vector. The number of new relations derived will be equal to the dimension of $\underline{\varphi}(t)$.

In this alternative of the Frisch method, two functions $\lambda_{y}\left(\lambda_{u}\right)$ of type (13) are evaluated, referring to the nominal model and the extended one, respectively. They correspond to two curves in the $\left(\lambda_{u}, \lambda_{y}\right)$ plane. The curves will ideally have one unique contact point, which defines the estimates, see Figure 1. See [2, 5] for details.

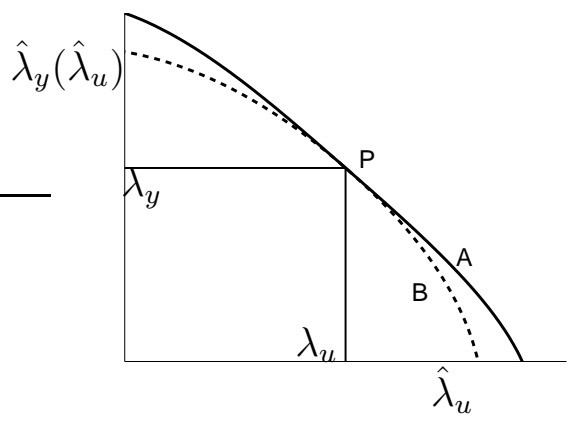

Figure 1: Illustration of the principle for the Frisch estimation using an extended model.

For the second alternative of the Frisch scheme, an additional relation is given as:

$$
\left.\frac{d}{d \lambda_{u}} V_{N}\left(\lambda_{u}\right)\right|_{\lambda_{u}=\hat{\lambda}_{u}}=0 .
$$

The estimated $\hat{\lambda}_{u}$ is determined as the minimizing element of the criterion

$$
\hat{\lambda}_{u}=\arg \min _{\lambda_{u}} V_{N}\left(\lambda_{u}\right) .
$$

The criterion $V_{N}\left(\hat{\lambda}_{u}\right)$ is defined as

$$
V_{N}\left(\hat{\lambda}_{u}\right)=\delta^{T} \Gamma \delta,
$$

where $\Gamma$ is a user chosen, positive definite weighting matrix. The vector $\delta$ is

$$
\delta=\left(\begin{array}{c}
\hat{r}_{\epsilon}(1)-\hat{r}_{\epsilon_{0}}(1) \\
\vdots \\
\hat{r}_{\epsilon}(m)-\hat{r}_{\epsilon_{0}}(m)
\end{array}\right)
$$


Note that $\hat{r}_{\epsilon}(0)-\hat{r}_{\epsilon_{0}}(0)$ is not used because it automatically equals to zero [12]. The maximum lag $m$ used in (19) is to be chosen by the user. In expression (19), $\hat{r}_{\epsilon}(k)$ are the sample covariance elements

$$
\hat{r}_{\epsilon}(k)=\frac{1}{N} \sum_{t=1}^{N} \epsilon(t, \hat{\theta}) \epsilon(t+k, \hat{\theta}) .
$$

where the residuals $\epsilon(t, \hat{\theta})$ are defined as

$$
\epsilon(t, \hat{\theta})=\hat{A}\left(q^{-1}\right) y(t)-\hat{B}\left(q^{-1}\right) u(t) .
$$

The theoretical covariance elements $\hat{r}_{\epsilon_{0}}(k)$ are based on the model

$$
\epsilon_{0}(t)=\hat{A}\left(q^{-1}\right) \hat{\tilde{y}}(t)-\hat{B}\left(q^{-1}\right) \hat{\tilde{u}}(t) .
$$

In the third variant of the Frisch scheme, the high order Yule-Walker equations are used [6]. Similar as the extended model alternative, a regressor vector is introduced as

$$
\underline{\varphi}(t)=\left(\begin{array}{lll}
u\left(t-n_{b}-1\right) & \ldots u\left(t-n_{b}-p\right)
\end{array}\right) .
$$

Because of Assumption A4 and equation (9), we get the following high order Yule-Walker equations

$$
\begin{aligned}
& \left(R_{\underline{\varphi} \phi}\right) \Theta=\mathbf{0} \\
\Leftrightarrow \quad & \left(R_{\underline{\varphi}_{0}} \phi_{0}\right) \Theta=\mathbf{0} .
\end{aligned}
$$

The noise variances $\lambda_{u}$ and $\lambda_{y}$ are then evaluated by searching the minimum of the cost function

$$
J\left(\lambda_{u}, \lambda_{y}\right)=\left\|R_{\underline{\varphi} \phi} \Theta\right\|^{2}=\Theta^{T} R_{\underline{\varphi} \phi}^{T} R_{\underline{\varphi} \phi} \Theta,
$$

with the constraint (13).

For convenience, in the following, the notations of Frisch-SR, Frisch-CM, and Frisch-YW will be used to represent the first, the second and the third alternative of the Frisch schemes, respectively.

We stress that there are different user choices for the three variants of Frisch. For Frisch-SR, we have freedom to choose the ways to extend the system model. Such as adding one A or B parameter, or several A and/or B parameters. If the extended model has only one additional parameter, then the number of equations is equal to the number of unknowns. If the extended model has more additional 
parameter, we will choose not only the parameters included in the extended vector, but also some possible weightings. For Frisch-CM, we will decide the number of the residual lags $m$. If $m>1$, we have overdetermined equations and the suitable weighting will also be chosen by us. Similarly, for Frisch-YW, the number and the type of the Yule-Walker equations and the possible weighting are the user choices. In general, for Frisch-CM and Frisch-YW, the number of equations is mostly larger than the number of the unknowns.

\section{Algorithmic aspects}

\subsection{Equations used in the Frisch schemes}

A recent analysis in [9] has shown that the equations used in Frisch-SR and in the BELS method [19] are equivalent when the same extended model is used. Under Assumption A4, the relations used in Frisch-SR equal to the following three equations

$$
\begin{gathered}
\left(R_{\varphi \varphi}-R_{\tilde{\varphi} \tilde{\varphi}}\right) \theta=r_{\varphi y}, \\
r_{y \varphi} \theta=r_{y}(0)-\lambda_{y}, \\
R_{\underline{\varphi} \varphi} \theta=r_{\underline{\varphi} y} .
\end{gathered}
$$

The first two equations (26) and (27) are coming from the basic Frisch equations (11) and (12), and equation (28) can be derived from equation (14), which is used in Frisch-SR. See [9] for a detailed proof.

In Frisch-YW, besides the basic Frisch equations (11) and (12), we use the equation (24), which can easily be further expressed as

$$
\begin{aligned}
& \left(R_{\underline{\varphi} \phi}\right) \Theta=\mathbf{0} \\
& \Leftrightarrow \quad\left(\begin{array}{ll}
-r_{\underline{\varphi} y} & R_{\underline{\varphi} \varphi}
\end{array}\right)\left(\begin{array}{l}
1 \\
\theta
\end{array}\right)=\mathbf{0} \\
& \Leftrightarrow \quad R_{\underline{\varphi} \varphi} \theta=r_{\underline{\varphi} y}
\end{aligned}
$$

i.e. equation (24) is also equivalent to equation (28). It follows that Frisch-SR and Frisch-YW are equivalent from the equations point of view providing that the same regressor vector $\underline{\varphi}(t)$ is used.

For the Frisch-CM, no explicit regressor vector $\underline{\varphi}(t)$ is used, and equation (16) can not be rewritten as (28). Frisch-CM is therefore different from Frisch-SR and Frisch-YW. 


\subsection{Algorithmic differences}

In all three Frisch methods we have a set of (overdetermined) nonlinear equations to solve. When choosing more than one additional parameters in the extended model, the lag number $m$ and the regressor vector size $p$ is larger than one, we will face the problem on how to handle the overdetermined equations in a good way. The statistically best way (in terms of covariance matrix of the parameter estimates) for the case of more equations than unknowns, is to solve all equations simultaneously in a weighted sense. The sets of nonlinear equations (26)-(28) or (26), (27), (16) can be written as

$$
f(\vartheta)=\mathbf{0}
$$

where

$$
\vartheta=\left(\theta^{T}, \theta_{\lambda}^{T}\right) \quad \theta_{\lambda}=\left(\lambda_{u}, \lambda_{y}\right)^{T} .
$$

Then the parameter vector $\vartheta$ can be estimated by

$$
\hat{\vartheta}=\arg \min _{\vartheta}\|f(\vartheta)\|_{W}^{2}=\arg \min _{\vartheta} f^{T}(\vartheta) W f(\vartheta),
$$

where $W$ is a positive definite weighting matrix designed by the user. There is an optimal choice of the weighting matrix, see [16], but the optimal weighting is computationally rather complex to derive explicitly.

In the described Frisch scheme methods, on the contrary, a different approach is followed. In fact, some of the equations are forced to hold exactly and some others approximately. This can be formulated as an optimization problem with equality constraints. In particular, the basic Frisch equations (11)-(12), must hold exactly. The remaining equations, that depend on the specific criterion, will hold approximately. For example, in the Frisch-CM method, if we choose

$$
W=\left(\begin{array}{cc}
\rho \mathbf{I} & \mathbf{0} \\
\mathbf{0} & \Gamma
\end{array}\right)
$$

and partition the original equations $f(\vartheta)$ into two parts, $f_{1}(\vartheta)$ and $f_{2}(\vartheta)$, then the loss function in (31) becomes

$$
f^{T}(\vartheta) W f(\vartheta)=\rho f_{1}^{T}(\vartheta) f_{1}(\vartheta)+f_{2}^{T}(\vartheta) \Gamma f_{2}(\vartheta) .
$$

If $\rho \rightarrow \infty$, it means that the first part of equations, $f_{1}(\vartheta)=0$, i.e. the basic Frisch equation (11), must hold exactly. The remaining equations, $f_{2}(\vartheta) \approx 0$, i.e. the additional equations (16), will hold approximately. 
The discussion in Section 3.1 concludes that the equations used in Frisch-SR and Frisch-YW are equivalent, while Frisch-CM is different. It means that the asymptotic statistical properties of Frisch-SR and Frisch-YW should be same but differ from Frisch-CM. However, the methods have different performances depending not only on the equations that they use but also on the techniques utilized for finding the solution. Even if all the Frisch algorithms can be reduced to a onedimensional search/one-dimensional optimisation problem, their computational complexities can be quite different, as shown by the simulation results in [5], [6]. Since the solution is searched within a well-defined locus [3], these algorithms do not suffer of convergence problems. Of course, they are not free from the risk of false minima in some critical conditions, such as quite low SNR and/or quite short number of data.

Also the different BELS variants suffer of the same type of problems. For example, the BELS method [19], which use a certain iterative algorithm, is not the best way to solve the set of equations. It can have convergence problems when the signal-to-noise ratio (SNR) is low. If the equations are solved using a variable projection algorithm then the performance will be improved [15].

\subsection{Possible extention to handle correlated noise}

Recently, the Frisch scheme was extended for identifying EIV systems with correlated output noise [14]. The additional equations were obtained by using the cross correlation of residuals and past input,

$$
\begin{array}{ll} 
& E\left(\begin{array}{c}
u\left(t-n_{b}-1\right) \\
\vdots \\
u\left(t-n_{b}-p\right)
\end{array}\right)\left(A\left(q^{-1}\right) y(t)-B\left(q^{-1}\right) u(t)\right)=\mathbf{0}, \\
\Leftrightarrow \quad & E \underline{\varphi}(t) \varepsilon(t)=\mathbf{0}, \\
\Leftrightarrow \quad & R_{\underline{\varphi} \varphi} \theta=r_{\underline{\varphi} y} .
\end{array}
$$

Further extentions to handle the case when both input and output sides have colored noises is more complicated. In this case, using the extended model, covariance matching and Yule-Walker equations to get additional equations do not work because the number of unknown noise parameters would be greater than the number of added equations. If the noise can be parametrically modeled as an ARMA process, the problem could be solved, however. Detailed descriptions are given in [7] and [9]. 


\section{Comparison and analysis of the asymptotic covari- ance matrices of the estimates}

For the three Frisch alternatives, it is of interest to know which one gives the best estimation accuracy. A statistical analysis of the accuracy can facilitate the evaluation and comparison of the methods. When the data number $N \rightarrow \infty$, an asymptotic covariance matrix of the parameter estimates is defined as

$$
P \triangleq \lim _{N \rightarrow \infty} E\left\{N \operatorname{cov}\left(\hat{\vartheta}-\vartheta_{0}\right)\left(\hat{\vartheta}-\vartheta_{0}\right)^{T}\right\}
$$

where $\hat{\vartheta}$ and $\vartheta_{0}$ denote the estimate and the true value of $\vartheta$, respectively.

Assume that $\hat{\vartheta}$ is close to the true parameter vector $\vartheta_{0}$ for large $N$. Then we linearize each equation used in the methods into the generic form

$$
\alpha_{\theta} \tilde{\theta}+\alpha_{\lambda_{u}} \tilde{\lambda}_{u}+\alpha_{\lambda_{y}} \tilde{\lambda}_{y} \approx \beta .
$$

The coefficients $\alpha_{\theta}, \alpha_{\lambda_{u}}, \alpha_{\lambda_{y}}$ are deterministic variables, while $\beta$ is a random term which has zero mean and a variance that decreases when $N$ increases.

Under the given assumptions in Section 2, the estimated parameter $\hat{\vartheta}$ is asymptotically Gaussian distributed

$$
\sqrt{N}\left(\hat{\vartheta}-\vartheta_{0}\right) \stackrel{\text { dist }}{\longrightarrow} \mathcal{N}(0, P),
$$

where

$$
\begin{aligned}
P & =\lim _{N \rightarrow \infty} N E\left\{\operatorname{cov}\left(\hat{\vartheta}-\vartheta_{0}\right)\left(\hat{\vartheta}-\vartheta_{0}\right)^{T}\right\} \\
& =\left(G^{T} W G\right)^{-1} G^{T} W Q W G\left(G^{T} W G\right)^{-1}
\end{aligned}
$$

The coefficients $\alpha_{\theta}, \alpha_{\lambda_{u}}, \alpha_{\lambda_{y}}$ appear as elements of $G$. The block elements of $Q$ are covariance matrices of the random terms $\beta$. A key step for realizing (38) is to consider $f(\vartheta)$ in (29) near the true value $\vartheta_{0}$ and approximate it as

$$
f(\vartheta)=f\left(\vartheta_{0}\right)+\frac{\partial f}{\partial \vartheta}\left(\hat{\vartheta}-\vartheta_{0}\right)=f\left(\vartheta_{0}\right)+G\left(\hat{\vartheta}-\vartheta_{0}\right) .
$$

If choosing the weighting matrix $W$ in (31) as

$$
W=Q^{-1},
$$

we will get the optimal minimal covariance matrix

$$
P_{\text {opt }}=\left(G^{T} W G\right)^{-1} \text {. }
$$


See [16] for a proof. However, this result is normally complicated to be utilized in practice, because the matrix $Q$, which is parameter $\vartheta$ related, needs to be known first before using (40).

The asymptotic covariance matrix $P$ of the estimates for Frisch-CM has been derived in [12]. For the equations used in Frisch-SR and Frisch-YW, we already proved that they are equivalent to each other and also equivalent to the equations used in BELS providing the same additional regressor vector $\underline{\varphi}(t)$ is used. If these equation sets are treated in the same way (using the same weighting etc.), the asymptotic covariance matrices of the Frish-SR and Frisch-YW methods will be identical to that of the BELS methods, which has been given in [9]. So we have the explicit expressions of the asymptotic covariance matrices for all three Frisch methods. For simplicity, only Gaussian data are considered here. The results could be extended to handle more general data as shown in [12] and [9].

\subsection{Numerical comparison of the asymptotic covariance ma- trices of Frisch methods under moderate SNR}

In this section, we use the asymptotic theoretical covariance matrices derived in [9] and [12] to numerically analyze the asymptotic estimation accuracy of the Frisch-SR and Frisch-CM methods by means of some examples. For the Frisch$\mathrm{SR}$, we choose the extended vector as $\underline{\varphi}(t)=-y\left(t-n_{a}-1\right)$. In Frisch-CM, the lag $m$ equals 5 and the weighting matrix $\Gamma$ is taken as in [4] and [12]:

$$
\Gamma=\left(\begin{array}{cccc}
2 m & 0 & \cdots & \\
0 & 2(m-1) & 0 & \\
& 0 & \ddots & \\
& & 0 & 2
\end{array}\right)
$$

Example 1: Consider a first-order system given by

$$
\left(1-0.8 q^{-1}\right) y_{0}(t)=2.0 q^{-1} u_{0}(t)
$$

The noise-free input $u_{0}(t)$ is the $\operatorname{ARMA}(1,1)$ process

$$
\left(1-0.5 q^{-1}\right) u_{0}(t)=\left(1+0.7 q^{-1}\right) e(t)
$$

where $e(t)$ is a zero-mean white noise with unit variance. The white measurement noises at the input and output sides have variance equals to 1 and 2, respectively. 
First for Frisch-SR with extended vector $\underline{\varphi}(t)=-y(t-2)$, the theoretical normalized asymptotic covariance matrix of the estimates is as follows

$$
P_{\text {Frisch-SR }}=\left(\begin{array}{rrrr}
0.068 & & & \\
0.299 & 3.701 & & \\
-0.458 & -5.438 & 35.25 & \\
0.161 & 1.921 & -4.743 & 5.229
\end{array}\right) \text {. }
$$

The corresponding normalized covariance matrix obtained from Frisch-CM is

$$
P_{\text {Frisch-CM }}=\left(\begin{array}{rrrr}
0.133 & & & \\
0.952 & 10.30 & & \\
-2.426 & -25.37 & 94.81 & \\
0.794 & 8.302 & -24.31 & 11.26
\end{array}\right) \text {. }
$$

We see in this example that the estimates of the Frisch-SR method is better (lower estimation error) than using the Frisch-CM method.

Example 2: The same system and the variances of the measurement noises as that in Example 1 were used. The noise-free input $u_{0}(t)$ was changed to white noise with unit variance. For Frisch-SR with extended vector as $\underline{\varphi}(t)=-y(t-2)$, the theoretical normalized asymptotic covariance matrix of the estimates is

$$
P_{\text {Frisch-SR }}=\left(\begin{array}{rrrr}
0.848 & & & \\
5.661 & 80.57 & & \\
-7.228 & -97.68 & 145.9 & \\
2.831 & 38.28 & -48.84 & 22.78
\end{array}\right) .
$$

The corresponding normalized covariance matrix obtained from the Frisch-CM method is

$$
P_{\text {Frisch-CM }}=\left(\begin{array}{rrrr}
0.736 & & & \\
3.598 & 47.61 & & \\
-4.612 & -56.62 & 94.81 & \\
1.511 & 18.53 & -24.31 & 11.26
\end{array}\right) \text {. }
$$

In this example, opposite to the results that we get in Example 1, the estimates of the Frisch-CM method are more accurate than those obtained using the Frisch-SR method. 
Example 3: Consider a second-order system

$$
\left(1-1.5 q^{-1}+0.7 q^{-2}\right) y_{0}(t)=\left(2.0 q^{-1}+1.0 q^{-2}\right) u_{0}(t),
$$

where $u_{0}(t)$ is the same as in Example 1, and the variances of the white measurement noises at the input and output sides are equal to 1 and 4, respectively. Assume $\varphi(t)=-y(t-3)$. Then the theoretical normalized asymptotic covariance matrix of the Frisch-SR scheme is

$$
P_{\text {Frisch-SR }}=\left(\begin{array}{rrrrrr}
0.33 & & & & & \\
-0.26 & 0.22 & & & & \\
-2.03 & 1.27 & 57.47 & & & \\
3.42 & -2.32 & -58.07 & 69.99 & & \\
-0.14 & 0.25 & -29.88 & 21.36 & 96.54 & \\
-0.07 & -0.01 & 14.75 & -11.51 & -14.49 & 11.36
\end{array}\right) \text {, }
$$

and Frisch-CM leads to

$$
P_{\text {Frisch-CM }}=\left(\begin{array}{rrrrrrr}
0.44 & & & & & \\
-0.34 & 0.28 & & & & \\
-2.36 & 1.59 & 46.6 & & & \\
4.45 & -3.17 & -52.5 & 73.3 & & \\
-0.68 & 0.63 & -22.1 & 11.6 & 97.1 & \\
0.29 & -0.26 & 7.22 & -3.34 & -13.0 & 9.0
\end{array}\right) \text {. }
$$

The results of this example show that, for some parameters, using Frisch-CM method gives better estimation accuracy than using Frisch-SR, while for some other parameters Frisch-SR works better instead.

Comparisons in the three examples above show that, when both input and output sides have moderate SNR, the accuracies of the Frisch-SR and Frisch-CM estimates differ for all the parameters. No alternative is always better than the other. Depending on the system, the noise free input signal, comparison criterion etc, one or the other version of the Frisch scheme may be considered to give the best result.

\subsection{Analysis of the asymptotic covariance matrices of Frisch methods under high SNR}

We will examine the accuracy properties of the three Frisch methods when both input and output SNR are high. Assume the noise free input $u_{0}(t)$ is an ARMA 
process

$$
u_{0}(t)=\frac{C\left(q^{-1}\right)}{D\left(q^{-1}\right)} e(t),
$$

where $e(t)$ is zero mean white noise with variance $\lambda_{e}$. Keeping $\lambda_{u}$ and $\lambda_{y}$ as constant and letting $\lambda_{e} \rightarrow \infty$, that is both input and output SNR tend to high values, we have the following two lemmas. For simplicity, no weighting is considered here.

Lemma 1: For Frisch-CM, the $G$ and $Q$ matrices in (38) can be partitioned, according to (30), as follows

$$
\begin{gathered}
G_{\mathrm{CM}}=\left(\begin{array}{cc}
\lambda_{e} M_{11} & M_{12} \\
\mathbf{0} & M_{22}
\end{array}\right), \\
Q_{\mathrm{CM}}=\left(\begin{array}{cc}
\lambda_{e} T_{11}+\tilde{T}_{11} & T_{12} \\
T_{21} & T_{22}
\end{array}\right),
\end{gathered}
$$

where all dependencies on $\lambda_{e}$ are as shown.

For the asymptotic covariance matrix $P$, which is expressed as

$$
P=\left(\begin{array}{ll}
P_{11} & P_{12} \\
P_{12}^{T} & P_{22}
\end{array}\right),
$$

it follows that $P_{11}$ depends on $\lambda_{e}$, and when $\lambda_{e}$ becomes very large, that is for (very) large SNR,

$$
\lim _{\lambda_{e} \rightarrow \infty}\left(\lambda_{e} P_{11}\right)=M_{11}^{-1} T_{11} M_{11}^{-T} .
$$

For $P_{12}$, it holds that

$$
\lambda_{e} P_{12}=M_{11}^{-1} T_{12} M_{22}^{-T}-M_{11}^{-1} M_{12} M_{22}^{-1} T_{22} M_{22}^{-T} .
$$

Furthermore, the block element $P_{22}$ does not depend at all on $\lambda_{e}$.

Proof: See Appendix A.

Lemma 2: For Frisch-SR and Frisch-YW, the $G$ and $Q$ matrices in (38) can be partitioned as

$$
G_{\mathrm{SR} / \mathrm{YW}}=\left(\begin{array}{cc}
\lambda_{e} M_{11} & M_{12} \\
\lambda_{e} M_{21} & M_{22}
\end{array}\right)
$$




$$
Q_{\mathrm{SR} / \mathrm{YW}}=\left(\begin{array}{cc}
\lambda_{e} T_{11}+\tilde{T}_{11} & \lambda_{e} T_{12}+\tilde{T}_{12} \\
\lambda_{e} T_{21}+\tilde{T}_{21} & \lambda_{e} T_{22}+\tilde{T}_{22}
\end{array}\right)
$$

(Note that the bock matrices $M_{i j}, T_{i j}$ and $\tilde{T}_{i j}$ are not the same in (47), (48) as in (52), (53).) It follows that the asymptotic covariance matrix $P$ equals

$$
P=\left(\begin{array}{ll}
P_{11} & P_{12} \\
P_{12}^{T} & P_{22}
\end{array}\right)
$$

where the block elements $P_{11}, P_{12}$ and $P_{22}$ all depend on $\lambda_{e}$. When $\lambda_{e}$ becomes very large, that is for (very) large SNR,

$$
\begin{aligned}
& \lim _{\lambda_{e} \rightarrow \infty}\left(\lambda_{e} P_{11}\right)=\left(V_{11} T_{11}+V_{12} T_{21}\right) V_{11}^{T}+\left(V_{11} T_{12}+V_{12} T_{22}\right) V_{12}^{T}, \\
& \lim _{\lambda_{e} \rightarrow \infty} P_{12}=\left(V_{11} T_{11}+V_{12} T_{21}\right) V_{21}^{T}+\left(V_{11} T_{12}+V_{12} T_{22}\right) V_{22}^{T}, \\
& \lim _{\lambda_{e} \rightarrow \infty} P_{22}=\infty
\end{aligned}
$$

where

$$
G^{-1}=\frac{1}{\lambda_{e}}\left(\begin{array}{cc}
V_{11} & V_{12} \\
\lambda_{e} V_{21} & \lambda_{e} V_{22}
\end{array}\right)
$$

Proof: See Appendix B.

We present an illustrative example to show the performance as stated by the preceding lemmas.

Example 4: Consider the same system as Example 1. We increased the variance of the noise-free input $\lambda_{e}$ from 1 to $10^{8}$ and kept the variances of the measurement noises $\lambda_{u}$ and $\lambda_{y}$ as 1 and 2, respectively. For the asymptotic covariance matrices of the estimated parameters by using Frisch-SR and Frisch-CM, their block elements $P_{11}, P_{12}$ and $P_{22}$ are listed in Table 1 and Table 2. For Frisch-SR, the values of the following items were calculated as

$$
\begin{aligned}
& M_{11}^{-1} T_{11} M_{11}^{-T}=\left(\begin{array}{cc}
5.78 e-02 & 1.74 e-01 \\
1.74 e-01 & 2.19 e+00
\end{array}\right) \\
& M_{11}^{-1} T_{12} M_{22}^{-T}-M_{11}^{-1} M_{12} M_{22}^{-1} T_{22} M_{22}^{-T}=\left(\begin{array}{ll}
-2.43 e+00 & 7.94 e-01 \\
-2.54 e+01 & 8.30 e+00
\end{array}\right), \\
& M_{22}^{-1} T_{22} M_{22}^{-T}=\left(\begin{array}{cc}
9.48 e+01 & -2.43 e+01 \\
-2.43 e+01 & 1.13 e+01
\end{array}\right) .
\end{aligned}
$$


And for Frisch-CM we have

$$
\begin{aligned}
& \left(V_{11} T_{11}+V_{12} T_{21}\right) V_{11}^{T}+\left(V_{11} T_{12}+V_{12} T_{22}\right) V_{12}^{T}=\left(\begin{array}{cc}
6.31 e-02 & 2.41 e-01 \\
2.41 e-01 & 3.0 e+00
\end{array}\right), \\
& \left(V_{11} T_{11}+V_{12} T_{21}\right) V_{21}^{T}+\left(V_{11} T_{12}+V_{12} T_{22}\right) V_{22}^{T}=\left(\begin{array}{cc}
-2.31 e-01 & 9.46 e-02 \\
-2.71 e+00 & 1.11 e+00
\end{array}\right) .
\end{aligned}
$$

We see that the equations (50)-(51) and (55)-(56) in Lemmas 1 and 2 are well supported by the the numerical results.

The preceeding analysis of the asymptotic covariance matrices of Frisch methods shows that the estimates for the system parameter $\theta$ for both Frisch-SR and Frisch-CM are good. The variances of the estimates decrease when the SNR increases and tends to a limit. The two limits of the estimates by Frisch-SR and Frisch-CM are different. For the noise parameters $\lambda_{u}$ and $\lambda_{y}$, in general, Frisch$\mathrm{CM}$ gives better estimates than Frisch-SR. In Frisch-CM, the estimates for the noise variances $\lambda_{u}$ and $\lambda_{y}$ keep the same accuracy when the SNR increases. In Frisch-SR, the variances of the estimates for $\lambda_{u}$ and $\lambda_{y}$ continuously increase with increasing SNR.

Table 1: The asymptotic covariance matrices of Example 4 with different SNR for Frisch-SR method. (Note: the blocks of the matrix, $P_{11}, P_{12}$ and $P_{22}$, are given.)

\begin{tabular}{r|rr|rr|rr}
\hline$\lambda_{e}$ & $P_{11}$ & $P_{12}$ & \multicolumn{2}{r}{$P_{22}$} \\
\hline 1 & $6.84 \mathrm{e}-02$ & $2.99 \mathrm{e}-01$ & $-4.58 \mathrm{e}-01$ & $1.61 \mathrm{e}-01$ & $3.52 \mathrm{e}+01$ & $-4.74 \mathrm{e}+00$ \\
& $2.99 \mathrm{e}-01$ & $3.70 \mathrm{e}+00$ & $-5.44 \mathrm{e}+00$ & $1.92 \mathrm{e}+00$ & $-4.74 \mathrm{e}+00$ & $5.23 \mathrm{e}+00$ \\
\hline 10 & $6.36 \mathrm{e}-03$ & $2.47 \mathrm{e}-02$ & $-2.53 \mathrm{e}-01$ & $1.01 \mathrm{e}-01$ & $1.14 \mathrm{e}+02$ & $-3.69 \mathrm{e}+01$ \\
& $2.47 \mathrm{e}-02$ & $3.07 \mathrm{e}-01$ & $-2.98 \mathrm{e}+00$ & $1.19 \mathrm{e}+00$ & $-3.69 \mathrm{e}+01$ & $1.85 \mathrm{e}+01$ \\
\hline 100 & $6.32 \mathrm{e}-04$ & $2.42 \mathrm{e}-03$ & $-2.33 \mathrm{e}-01$ & $9.53 \mathrm{e}-02$ & $8.98 \mathrm{e}+02$ & $-3.58 \mathrm{e}+02$ \\
& $2.42 \mathrm{e}-03$ & $3.01 \mathrm{e}-02$ & $-2.73 \mathrm{e}+00$ & $1.12 \mathrm{e}+00$ & $-3.58 \mathrm{e}+02$ & $1.50 \mathrm{e}+02$ \\
\hline 1000 & $6.31 \mathrm{e}-05$ & $2.41 \mathrm{e}-04$ & $-2.31 \mathrm{e}-01$ & $9.47 \mathrm{e}-02$ & $8.74 \mathrm{e}+03$ & $-3.57 \mathrm{e}+03$ \\
& $2.41 \mathrm{e}-04$ & $3.00 \mathrm{e}-03$ & $-2.71 \mathrm{e}+00$ & $1.11 \mathrm{e}+00$ & $-3.57 \mathrm{e}+03$ & $1.47 \mathrm{e}+03$ \\
\hline $1 \mathrm{e}+08$ & $6.31 \mathrm{e}-10$ & $2.41 \mathrm{e}-09$ & $-2.31 \mathrm{e}-01$ & $9.46 \mathrm{e}-02$ & $8.71 \mathrm{e}+08$ & $-3.57 \mathrm{e}+08$ \\
& $2.41 \mathrm{e}-09$ & $3.04 \mathrm{e}-08$ & $-2.71 \mathrm{e}+00$ & $1.11 \mathrm{e}+00$ & $-3.57 \mathrm{e}+08$ & $1.46 \mathrm{e}+08$ \\
\hline
\end{tabular}

\section{Comparison of the Frisch methods under finite data}

In this section, a comparison of the three Frisch criteria in presence of finite data is performed by means of some Monte Carlo simulations. 
Table 2: The asymptotic covariance matrices of Example 4 with different SNR for Frisch-CM method. (Note: the blocks of the matrix, $P_{11}, P_{12}$ and $P_{22}$, are given.)

\begin{tabular}{r|rr|rr|rr}
\hline$\lambda_{e}$ & $P_{11}$ & $P_{12}$ & \multicolumn{2}{r}{$P_{22}$} \\
\hline 1 & $1.33 \mathrm{e}-01$ & $9.52 \mathrm{e}-01$ & $-2.43 \mathrm{e}+00$ & $7.94 \mathrm{e}-01$ & $9.48 \mathrm{e}+01$ & $-2.43 \mathrm{e}+01$ \\
& $9.52 \mathrm{e}-01$ & $1.03 \mathrm{e}+01$ & $-2.54 \mathrm{e}+01$ & $8.30 \mathrm{e}+00$ & $-2.43 \mathrm{e}+01$ & $1.13 \mathrm{e}+01$ \\
\hline 10 & $6.52 \mathrm{e}-03$ & $2.52 \mathrm{e}-02$ & $-2.43 \mathrm{e}-01$ & $7.94 \mathrm{e}-02$ & $9.48 \mathrm{e}+01$ & $-2.43 \mathrm{e}+01$ \\
& $2.52 \mathrm{e}-02$ & $3.00 \mathrm{e}-01$ & $-2.54 \mathrm{e}+00$ & $8.30 \mathrm{e}-01$ & $-2.43 \mathrm{e}+01$ & $1.13 \mathrm{e}+01$ \\
\hline 100 & $5.85 \mathrm{e}-04$ & $1.82 \mathrm{e}-03$ & $-2.43 \mathrm{e}-02$ & $7.94 \mathrm{e}-03$ & $9.48 \mathrm{e}+01$ & $-2.43 \mathrm{e}+01$ \\
& $1.82 \mathrm{e}-03$ & $2.27 \mathrm{e}-02$ & $-2.54 \mathrm{e}-01$ & $8.30 \mathrm{e}-02$ & $-2.43 \mathrm{e}+01$ & $1.13 \mathrm{e}+01$ \\
\hline 1000 & $5.79 \mathrm{e}-05$ & $1.75 \mathrm{e}-04$ & $-2.43 \mathrm{e}-03$ & $7.94 \mathrm{e}-04$ & $9.48 \mathrm{e}+01$ & $-2.43 \mathrm{e}+01$ \\
& $1.75 \mathrm{e}-04$ & $2.20 \mathrm{e}-03$ & $-2.54 \mathrm{e}-02$ & $8.30 \mathrm{e}-03$ & $-2.43 \mathrm{e}+01$ & $1.13 \mathrm{e}+01$ \\
\hline $1 \mathrm{e}+08$ & $5.78 \mathrm{e}-10$ & $1.74 \mathrm{e}-09$ & $-2.43 \mathrm{e}-08$ & $7.94 \mathrm{e}-09$ & $9.48 \mathrm{e}+01$ & $-2.43 \mathrm{e}+01$ \\
& $1.74 \mathrm{e}-09$ & $2.19 \mathrm{e}-08$ & $-2.54 \mathrm{e}-07$ & $8.30 \mathrm{e}-08$ & $-2.43 \mathrm{e}+01$ & $1.13 \mathrm{e}+01$ \\
\hline
\end{tabular}

Example 5: The same system and the same variances of Example 3 have been used. We will assume $\underline{\varphi}(t)=-y(t-3)$ for both Frisch-SR and Frisch-YW. The normalized root mean square error

$$
\operatorname{NRMSE}=\frac{1}{\left\|\vartheta_{0}(i)\right\|} \sqrt{\frac{1}{M} \sum_{i=1}^{M}\left\|\hat{\vartheta}^{k}(i)-\vartheta_{0}(i)\right\|^{2}}
$$

has been used as performance index of the estimation, where $\hat{\vartheta}^{k}(i)$ denotes the estimate of the $i$-th element of $\vartheta_{0}$ obtained in the $k$-th run of the Monte Carlo simulation while $M$ is the number of runs. The methods have been compared by considering the following number of samples: $N=100,300,500,750,1000$. For each value of $N$ a Monte Carlo simulation of $M=300$ independent runs has been performed using Gaussian noise sequences. The NRMSEs of the estimated system parameters and noise variances are reported in Figures 2 and 3. It can be noted that, even though the behaviour of the methods under finite data can differ from the asymptotic one, the obtained results lead to the same conclusions as in Section 4 , that is no criterion is always better than the others.

\section{Conclusions}

The report has compared the estimation accuracy of three different Frisch criteria on the basis of the asymptotic covariance matrices of the estimates. The theoretical results have been supported by some numerical examples. It has been 

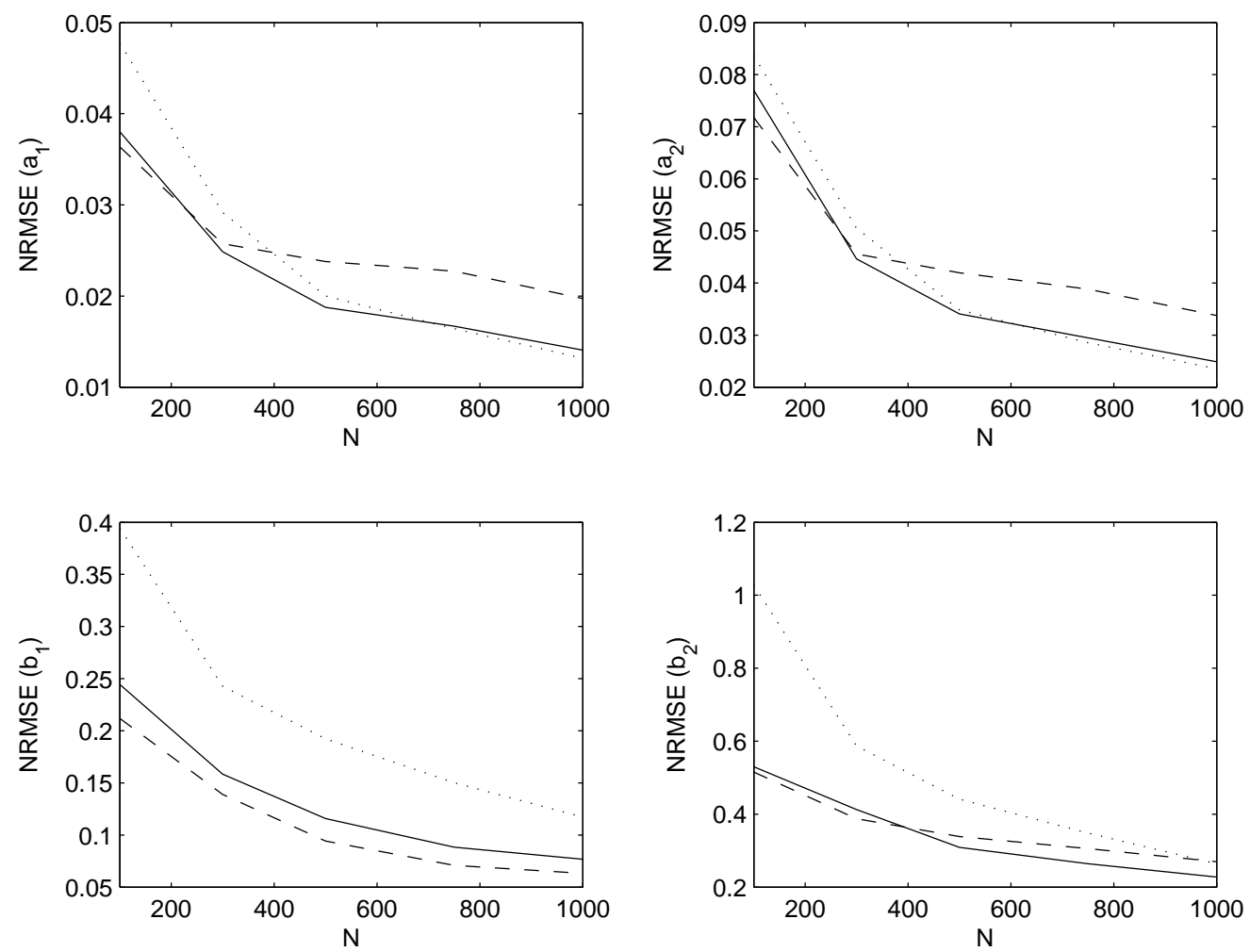

Figure 2: Normalized root mean square errors of the estimated system parameters versus the number of samples: Frisch-SR (solid), Frisch-CM (dashed), FrischYW (dotted). For every value of $N$ a Monte Carlo simulation of 300 runs has been performed.

shown that for moderate signal-to-noise ratios (SNR) no alternative is better than the other, both for the system parameters and noise variances. On the contrary, for high SNR the noise variances can, in general, be better estimated when the Frisch-CM criterion is used. Finally, some Monte Carlo simulations have been performed to compare the behaviour of the proposed methods under finite data.

\section{Acknowledgment:}

This research was partially supported by The Swedish Research Council, contract 621-2005-4207, and The Italian Ministry for University and Research. 

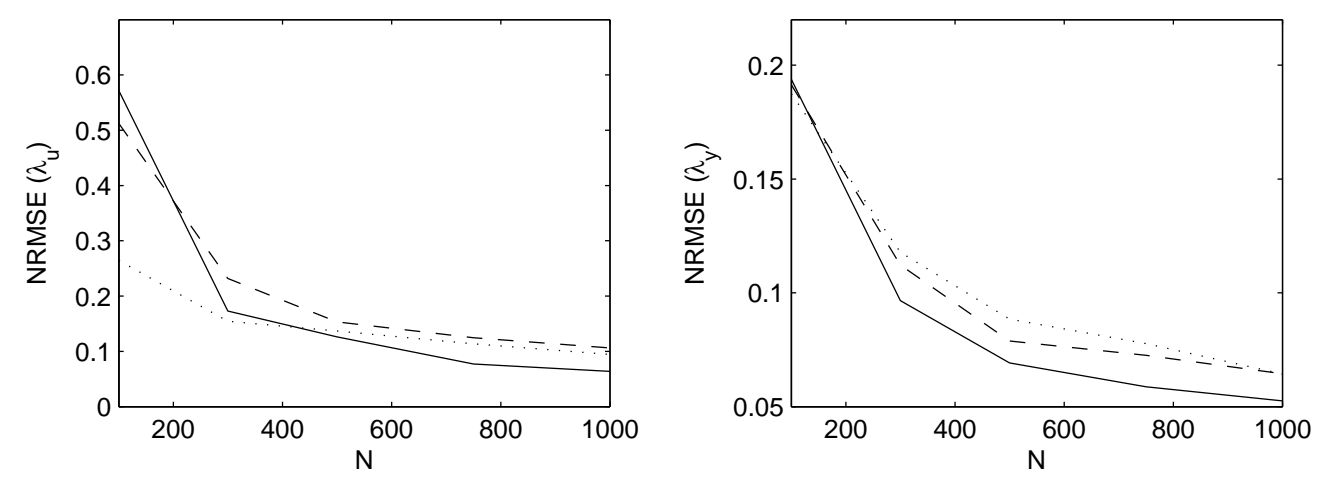

Figure 3: Normalized root mean square errors of the estimated noise variances versus the number of samples: Frisch-SR (solid), Frisch-CM (dashed), FrischYW (dotted). For every value of $N$ a Monte Carlo simulation of 300 runs has been performed.

\section{A Proof of Lemma 1}

From Section 2 and 3, we know that the Frisch-CM algorithm consists of the equations (26), (13) and (16). By linearizing each equation, the asymptotic covariance matrix can be expressed explicitly as formula (38) by inserting corresponding $G$ and $Q$ matrices. Here no weighting is considered, i.e. $W=I$.

Introduce the conventions

$$
\begin{aligned}
a_{i} & =\left\{\begin{array}{ll}
1 & i=0 \\
0 & i>n_{a}, i<0
\end{array},\right. \\
b_{i} & =0 \quad i>n_{b}, i \leq 0,
\end{aligned}
$$

and the vector

$$
\gamma=W \beta
$$

where the vector $\beta$ is given elementwise as

$$
\beta_{k}=-\sum_{i} b_{i} b_{i+k}+\sum_{i} a_{i} a_{i+k} \frac{\mathbf{b}^{T} \mathbf{b}}{\overline{\mathbf{a}}^{T} \overline{\mathbf{a}}}, \quad k=1, \ldots, m .
$$

For the Frisch-CM methods, the matrix $G$ and the symmetric matrix $Q$ are expressed as:

$$
G=\left(\begin{array}{ccc}
R_{\varphi_{0} \varphi_{0}} & \left(\begin{array}{c}
\mathbf{0} \\
-\mathbf{b}
\end{array}\right) & \left(\begin{array}{c}
-\mathbf{a} \\
\mathbf{0}
\end{array}\right) \\
\mathbf{0} & \mathbf{b}^{T} \mathbf{b} & \overline{\mathbf{a}}^{T} \overline{\mathbf{a}} \\
\mathbf{0} & \sum_{k=1}^{m} \gamma_{k} \sum_{i} b_{i} b_{i+k} & \sum_{k=1}^{m} \gamma_{k} \sum_{i} a_{i} a_{i+k}
\end{array}\right),
$$




$$
Q=\left(\begin{array}{lll}
Q_{11} & Q_{12} & Q_{13} \\
Q_{21} & Q_{22} & Q_{23} \\
Q_{31} & Q_{32} & Q_{33}
\end{array}\right)
$$

The blocks of the symmetric matrix $Q$ are as follows

$$
\begin{aligned}
Q_{11} & =\sum_{\tau} R_{\varphi_{0} \varphi_{0}}(\tau) r_{\varepsilon \varepsilon}(\tau)+\sum_{\tau}\left[R_{\tilde{\varphi} \tilde{\varphi}}(\tau) r_{\varepsilon \varepsilon}(\tau)+r_{\tilde{\varphi} \varepsilon}(\tau) r_{\tilde{\varphi} \varepsilon}^{T}(-\tau)\right] \\
Q_{12} & =2 \sum_{\tau} r_{\tilde{\varphi} \varepsilon}(\tau) r_{\varepsilon \varepsilon}(\tau) \\
Q_{13} & =\sum_{k=1}^{m} \gamma_{k}\left\{\sum_{\tau}\left[r_{\tilde{\varphi} \varepsilon}(\tau) r_{\varepsilon \varepsilon}(\tau-k)+r_{\tilde{\varphi} \varepsilon}(\tau-k) r_{\varepsilon \varepsilon}(\tau)\right]\right\} \\
Q_{22} & =2 \sum_{\tau} r_{\varepsilon \varepsilon}^{2}(\tau) \\
Q_{23} & =2 \sum_{k=1}^{m} \gamma_{k} \sum_{\tau}\left[r_{\varepsilon \varepsilon}(\tau) r_{\varepsilon \varepsilon}(\tau-k)\right] \\
Q_{33} & =\sum_{k=1}^{m} \sum_{j=1}^{m} \gamma_{k} \gamma_{j} \sum_{\tau}\left[r_{\varepsilon \varepsilon}(\tau) r_{\varepsilon \varepsilon}(\tau+k-j)+r_{\varepsilon \varepsilon}(\tau-j) r_{\varepsilon \varepsilon}(\tau+k)\right]
\end{aligned}
$$

See Appendix in [12] for a detailed proof.

According to assumption A4, the matrices $G$ and $Q$ can be expressed in partitioned form as

$$
G=\left(\begin{array}{cc}
\lambda_{e} M_{11} & M_{12} \\
\mathbf{0} & M_{22}
\end{array}\right), \quad Q=\left(\begin{array}{cc}
\lambda_{e} T_{11}+\tilde{T}_{11} & T_{12} \\
T_{21} & T_{22}
\end{array}\right),
$$

with

$$
\begin{aligned}
M_{11} & =\frac{1}{\lambda_{e}} R_{\varphi_{0} \varphi_{0}}, \quad M_{12}=\left(\begin{array}{cc}
\mathbf{0} & -\mathbf{a} \\
-\mathbf{b} & \mathbf{0}
\end{array}\right), \\
M_{22} & =\left(\begin{array}{cc}
\sum_{k=1}^{m} \gamma_{k} \sum_{i} b_{i} b_{i+k} & \sum_{k=1}^{m} \gamma_{k} \sum_{i} a_{i} a_{i+k}
\end{array}\right) \\
T_{11} & =\frac{1}{\lambda_{e}} \sum_{\tau} R_{\varphi_{0} \varphi_{0}}(\tau) r_{\varepsilon \varepsilon}(\tau), \quad \tilde{T}_{11}=\sum_{\tau}\left(R_{\tilde{\varphi} \tilde{\varphi}}(\tau) r_{\varepsilon \varepsilon}(\tau)+r_{\tilde{\varphi} \varepsilon}(\tau) r_{\tilde{\varphi} \varepsilon}^{T}(-\tau)\right), \\
T_{12} & =\left(\begin{array}{ll}
Q_{12} & Q_{13}
\end{array}\right), \quad T_{21}=\left(\begin{array}{c}
Q_{21} \\
Q_{31}
\end{array}\right), \quad T_{22}=\left(\begin{array}{ll}
Q_{22} & Q_{23} \\
Q_{32} & Q_{33}
\end{array}\right) .
\end{aligned}
$$

According to equation (38), when $W=I$, the asymptotic covariance matrix $P$ equals

$$
P=G^{-1} Q G^{-T}
$$


Inserting (72) into (73) gives

$$
P=\left(\begin{array}{ll}
P_{11} & P_{12} \\
P_{12}^{T} & P_{22}
\end{array}\right),
$$

with

$$
\begin{aligned}
P_{11}= & \frac{1}{\lambda_{e}} M_{11}^{-1} T_{11} M_{11}^{-T}+\frac{1}{\lambda_{e}^{2}}\left(M_{11}^{-1} \tilde{T}_{11} M_{11}^{-T}+M_{11}^{-1} M_{12} M_{22}^{-1} T_{22} M_{22}^{-T} M_{12}^{T} M_{11}^{-T}\right. \\
& \left.-M_{11}^{-1} T_{12} M_{22}^{-T} M_{12}^{T} M_{11}^{-T}-M_{11}^{-1} M_{12} M_{22}^{-1} T_{21} M_{11}^{-T}\right) \\
P_{12}= & \frac{1}{\lambda_{e}}\left(M_{11}^{-1} T_{12} M_{22}^{-T}-M_{11}^{-1} M_{12} M_{22}^{-1} T_{22} M_{22}^{-T}\right) \\
P_{22}= & M_{22}^{-1} T_{22} M_{22}^{-T} .
\end{aligned}
$$

It follows that

$$
\begin{aligned}
& \lim _{\lambda_{e} \rightarrow \infty}\left(\lambda_{e} P_{11}\right)=M_{11}^{-1} T_{11} M_{11}^{-T}, \\
& \lambda_{e} P_{12}=M_{11}^{-1} T_{12} M_{22}^{-T}-M_{11}^{-1} M_{12} M_{22}^{-1} T_{22} M_{22}^{-T},
\end{aligned}
$$

and $P_{22}$ does not relate to $\lambda_{e}$. Hence Lemma 1 follows.

\section{B Proof of Lemma 2}

In Section 3 and in paper [9], we showed that both Frisch-SR and Frisch-YW consist of equations (26)-(28). Linearization result of equation (26) has already been given in [10] Appendix A. Utilizing the same technique, the linearization of equations (27) and (28) are given as:

$$
\begin{gathered}
R_{y_{0} \varphi_{0}} \tilde{\theta}+\tilde{\lambda}_{y} \approx \frac{1}{N} \sum y(t) \varepsilon(t)-E y(t) \varepsilon(t) . \\
R_{\underline{\varphi}_{0} \varphi_{0}} \tilde{\theta} \approx \frac{1}{N} \sum \underline{\varphi}(t) \varepsilon(t)-E \underline{\varphi}(t) \varepsilon(t) .
\end{gathered}
$$

Then for the Frisch-SR and Frisch-YW methods, the matrix $G$ is

$$
G=\left(\begin{array}{ccc}
R_{\varphi_{0}} & \left(\begin{array}{c}
\mathbf{0} \\
-\mathbf{b}
\end{array}\right) & \left(\begin{array}{c}
-\mathbf{a} \\
\mathbf{0}
\end{array}\right) \\
R_{y_{0} \varphi_{0}} & 0 & 1 \\
R_{\underline{\varphi}_{0} \varphi_{0}} & \mathbf{0} & \mathbf{0}
\end{array}\right)
$$


and the blocks in the symmetric matrix $Q$ are expressed as:

$$
\begin{aligned}
Q_{11} & =\sum_{\tau}\left(r_{\varphi \varepsilon}(\tau) r_{\varphi \varepsilon}^{T}(-\tau)+R_{\varphi \varphi}(\tau) r_{\varepsilon \varepsilon}(\tau)\right) \\
Q_{12} & =\sum_{\tau}\left(r_{\varphi y}(\tau) r_{\varepsilon \varepsilon}(\tau)+r_{\varphi \varepsilon}(\tau) r_{\varepsilon y}(\tau)\right) \\
Q_{22} & =\sum_{\tau}\left(r_{y \varepsilon}(\tau) r_{\varepsilon y}(\tau)+r_{y y}(\tau) r_{\varepsilon \varepsilon}(\tau)\right) \\
Q_{13} & =\sum_{\tau}\left(r_{\varphi \varepsilon}(\tau) r_{\underline{\varphi} \varepsilon}^{T}(-\tau)+R_{\varphi \underline{\varphi}}(\tau) r_{\varepsilon \varepsilon}(\tau)\right) \\
Q_{23} & =\sum_{\tau}\left(r_{\underline{\varphi} y}(\tau) r_{\varepsilon \varepsilon}(\tau)+r_{\underline{\varphi \varepsilon}}(\tau) r_{\varepsilon y}(\tau)\right) \\
Q_{33} & =\sum_{\tau}\left(r_{\underline{\varphi \varepsilon}}(\tau) r_{\underline{\varphi} \varepsilon}^{T}(-\tau)+R_{\underline{\varphi \varphi}}(\tau) r_{\varepsilon \varepsilon}(\tau)\right) .
\end{aligned}
$$

From the assumption A4, each block of $Q$ can be further separated as one part depending on $\lambda_{e}$ and another part not related to $\lambda_{e}$, as shown in the following

$$
\begin{aligned}
Q_{11} & =\sum_{\tau} R_{\varphi_{0} \varphi_{0}}(\tau) r_{\varepsilon \varepsilon}(\tau)+\sum_{\tau}\left(r_{\tilde{\varphi} \varepsilon}(\tau) r_{\tilde{\varphi} \varepsilon}^{T}(-\tau)+R_{\tilde{\varphi} \tilde{\varphi}}(\tau) r_{\varepsilon \varepsilon}(\tau)\right) \\
& \triangleq \lambda_{e} Q_{0}^{11}+\tilde{Q}^{11}, \\
Q_{12} & =\sum_{\tau} r_{\varphi_{0} y_{0}}(\tau) r_{\varepsilon \varepsilon}(\tau)+\sum_{\tau}\left(r_{\tilde{\varphi} \tilde{y}}(\tau) r_{\varepsilon \varepsilon}(\tau)+r_{\tilde{\varphi} \varepsilon}(\tau) r_{\varepsilon \tilde{y}}(\tau)\right) \\
& \triangleq \lambda_{e} Q_{0}^{12}+\tilde{Q}^{12}, \\
Q_{22} & =\sum_{\tau} r_{y_{0} y_{0}}(\tau) r_{\varepsilon \varepsilon}(\tau)+\sum_{\tau}\left(r_{\tilde{y} \tilde{y}}(\tau) r_{\varepsilon \varepsilon}(\tau)+r_{\tilde{y} \varepsilon}(\tau) r_{\varepsilon \tilde{y}}(\tau)\right), \\
& \triangleq \lambda_{e} Q_{0}^{22}+\tilde{Q}^{22}, \\
Q_{13} & =\sum_{\tau} R_{\varphi_{0} \underline{\varphi}_{0}}(\tau) r_{\varepsilon \varepsilon}(\tau)+\sum_{\tau}\left(r_{\tilde{\varphi} \varepsilon}(\tau) r_{\tilde{\varphi} \varepsilon}^{T}(-\tau)+R_{\tilde{\varphi} \tilde{\underline{\varphi}}}(\tau) r_{\varepsilon \varepsilon}(\tau)\right), \\
& \triangleq \lambda_{e} Q_{0}^{13}+\tilde{Q}^{13}, \\
Q_{23} & =\sum_{\tau} r_{\underline{\varphi}_{0} y_{0}}(\tau) r_{\varepsilon \varepsilon}(\tau)+\sum_{\tau}\left(r_{\tilde{\tilde{y}} \tilde{y}}(\tau) r_{\varepsilon \varepsilon}(\tau)+r_{\underline{\tilde{\varphi} \varepsilon}}(\tau) r_{\varepsilon \tilde{y}}(\tau)\right), \\
& \triangleq \lambda_{e} Q_{0}^{23}+\tilde{Q}^{23}, \\
Q_{33} & =\sum_{\tau} R_{\underline{\varphi}_{0} \underline{\varphi}_{0}}(\tau) r_{\varepsilon \varepsilon}(\tau)+\sum_{\tau}\left(r_{\tilde{\tilde{\varphi} \varepsilon}}(\tau) r_{\underline{\tilde{\varphi} \varepsilon}}^{T}(-\tau)+R_{\underline{\tilde{\varphi} \tilde{\varphi}}}(\tau) r_{\varepsilon \varepsilon}(\tau)\right), \\
& \triangleq \lambda_{e} Q_{0}^{33}+\tilde{Q}^{33},
\end{aligned}
$$


Then the matrices $G$ and $Q$ in (38) can be expressed as

$$
G=\left(\begin{array}{cc}
\lambda_{e} M_{11} & M_{12} \\
\lambda_{e} M_{21} & M_{22}
\end{array}\right), \quad Q=\lambda_{e}\left(\begin{array}{ll}
T_{11} & T_{12} \\
T_{21} & T_{22}
\end{array}\right)+\left(\begin{array}{cc}
\tilde{T}_{11} & \tilde{T}_{12} \\
\tilde{T}_{21} & \tilde{T}_{22}
\end{array}\right)
$$

with

$$
\begin{aligned}
M_{11} & =\frac{1}{\lambda_{e}} R_{\varphi_{0} \varphi_{0}}, \quad M_{12}=\left(\begin{array}{cc}
\mathbf{0} & -\mathbf{a} \\
-\mathbf{b} & \mathbf{0}
\end{array}\right), \\
M_{21} & =\frac{1}{\lambda_{e}}\left(\begin{array}{l}
R_{y_{0} \varphi_{0}} \\
R_{\underline{\varphi}_{0} \varphi_{0}}
\end{array}\right), \quad M_{22}=\left(\begin{array}{ll}
0 & 1 \\
\mathbf{0} & \mathbf{0}
\end{array}\right) \\
T_{11} & =Q_{0}^{11}, \quad T_{12}=\left(\begin{array}{ll}
Q_{0}^{12} & Q_{0}^{13}
\end{array}\right), \\
T_{21} & =\left(\begin{array}{l}
Q_{0}^{21} \\
Q_{0}^{31}
\end{array}\right), \quad T_{22}=\left(\begin{array}{ll}
Q_{0}^{22} & Q_{0}^{23} \\
Q_{0}^{32} & Q_{0}^{33}
\end{array}\right), \\
\tilde{T}_{11} & =\tilde{Q}^{11}, \quad \tilde{T}_{12}=\left(\begin{array}{ll}
\tilde{Q}^{12} & \tilde{Q}^{13}
\end{array}\right), \\
\tilde{T}_{21} & =\left(\begin{array}{l}
\tilde{Q}^{21} \\
\tilde{Q}^{31}
\end{array}\right), \quad \tilde{T}_{22}=\left(\begin{array}{ll}
\tilde{Q}^{22} & \tilde{Q}^{23} \\
\tilde{Q}^{32} & \tilde{Q}^{33}
\end{array}\right) .
\end{aligned}
$$

Write the inverse of $G$ as

$$
G^{-1}=\frac{1}{\lambda_{e}}\left(\begin{array}{cc}
V_{11} & V_{12} \\
\lambda_{e} V_{21} & \lambda_{e} V_{22}
\end{array}\right),
$$

where

$$
\begin{aligned}
& V_{11}=M_{11}^{-1}+M_{11}^{-1} M_{12}\left(M_{22}-M_{21} M_{11}^{-1} M_{12}\right)^{-1} M_{21} M_{11}^{-1} \\
& V_{12}=-M_{11}^{-1} M_{12}\left(M_{22}-M_{21} M_{11}^{-1} M_{12}\right)^{-1} \\
& V_{21}=-\left(M_{22}-M_{21} M_{11}^{-1} M_{12}\right)^{-1} M_{21} M_{11}^{-1} \\
& V_{22}=\left(M_{22}-M_{21} M_{11}^{-1} M_{12}\right)^{-1}
\end{aligned}
$$

Similarly as in Appendix A, insert (87) into (73). This gives the asymptotic covariance matrix $P$ as

$$
P=\left(\begin{array}{ll}
P_{11} & P_{12} \\
P_{12}^{T} & P_{22}
\end{array}\right)
$$

with

$$
\begin{aligned}
P_{11}= & \frac{1}{\lambda_{e}}\left(\left(V_{11} T_{11}+V_{12} T_{21}\right) V_{11}^{T}+\left(V_{11} T_{12}+V_{12} T_{22}\right) V_{12}^{T}\right) \\
& +\frac{1}{\lambda_{e}^{2}}\left(\left(V_{11} \tilde{T}_{11}+V_{12} \tilde{T}_{21}\right) V_{11}^{T}+\left(V_{11} \tilde{T}_{12}+V_{12} \tilde{T}_{22}\right) V_{12}^{T}\right),
\end{aligned}
$$




$$
\begin{aligned}
P_{12}= & \left(\left(V_{11} T_{11}+V_{12} T_{21}\right) V_{21}^{T}+\left(V_{11} T_{12}+V_{12} T_{22}\right) V_{22}^{T}\right) \\
& +\frac{1}{\lambda_{e}}\left(\left(V_{11} \tilde{T}_{11}+V_{12} \tilde{T}_{21}\right) V_{21}^{T}+\left(V_{11} \tilde{T}_{12}+V_{12} \tilde{T}_{22}\right) V_{22}^{T}\right), \\
P_{22}= & \lambda_{e}\left(\left(V_{21} T_{11}+V_{22} T_{21}\right) V_{21}^{T}+\left(V_{21} T_{12}+V_{22} T_{22}\right) V_{22}^{T}\right) \\
& +\left(\left(V_{21} \tilde{T}_{11}+V_{22} \tilde{T}_{21}\right) V_{21}^{T}+\left(V_{21} \tilde{T}_{12}+V_{22} \tilde{T}_{22}\right) V_{22}^{T}\right) .
\end{aligned}
$$

From relations (94)-(96), we have

$$
\begin{aligned}
& \lim _{\lambda_{e} \rightarrow \infty}\left(\lambda_{e} P_{11}\right)=\left(V_{11} T_{11}+V_{12} T_{21}\right) V_{11}^{T}+\left(V_{11} T_{12}+V_{12} T_{22}\right) V_{12}^{T}, \\
& \lim _{\lambda_{e} \rightarrow \infty} P_{12}=\left(V_{11} T_{11}+V_{12} T_{21}\right) V_{21}^{T}+\left(V_{11} T_{12}+V_{12} T_{22}\right) V_{22}^{T}, \\
& \lim _{\lambda_{e} \rightarrow \infty} P_{22}=\infty
\end{aligned}
$$

and Lemma 2 follows.

\section{References}

[1] M. Aoki and P. C. Yue. On a priori error estimate of some identification methods. IEEE Transactions on Automatic Control, AC-15:541-548, October 1970.

[2] S. Beghelli, P. Castaldi, R. Guidorzi, and U. Soverini. A robust criterion for model selection in identification from noisy data. In Proc. 9th International Conference on Systems Engineering, pages 480-484, Las Vegas, Nevada, USA, 1993.

[3] S. Beghelli, R.P. Guidorzi, and U. Soverini. The Frisch scheme in dynamic system identification. Automatica, 26:171-176, 1990.

[4] R. Diversi, R. Guidorzi, and U. Soverini. A new criterion in EIV identification and filtering applications. In Proc. 13th IFAC Symposium on System Identification, pages 1993-1998, Rotterdam, The Netherlands, August 27-29 2003.

[5] R. Diversi, R. Guidorzi, and U. Soverini. Frisch scheme-based algorithms for eiv identification. In Proc. 12th IEEE Mediterranean Conference on Control and Automation, Kusadasi, Turkey, June 2004. 
[6] R. Diversi, U. Soverini, and R. Guidorzi. Yule-walker equations in the frisch scheme solution of errors-in-variables identification problems. In Proc. 17th International Symposium on Mathematical Theory of Networks and Systems (MTNS), pages 391-395, Kyoto, Japan, July 2006.

[7] M. Ekman, M. Hong, and T. Söderström. A separable nonlinear least squares approach for identification of linear systems with errors in variables. In Proc. 14th IFAC Symposium on System Identification, Newcastle, Australia, March 29-31 2006.

[8] R. Frisch. Statistical confluence analysis by means of complete regression systems. Technical Report 5, University of Oslo, Economics Institute, Oslo, Norway, 1934.

[9] M. Hong and T. Söderström. Relations between bias-eliminating least squares, the frisch scheme and extended compensated least squares methods for identifying errors-in-variables systems. 2007. Submitted.

[10] M. Hong, T. Söderström, and W. X. Zheng. Accuray analysis of biaseliminating least squares estimates for errors-in-variables systems. Automatica, 43(9):1590-1596, September 2007.

[11] M. J. Levin. Estimation of a system pulse transfer function in the presence of noise. IEEE Transactions on Automatic Control, AC-9:229-235, July 1964.

[12] T. Söderström. Accuracy analysis of the Frisch estimates for identifying errors-in-variables systems. IEEE Transactions on Automatic Control, 52(6):985-987, June 2007.

[13] T. Söderström. Errors-in-variables methods in system identification. Automatica, 43(6):939-958, June 2007. Survey paper.

[14] T. Söderström. Extending the Frisch scheme for errors-in-variables identification to correlated noise. International Journal of Adaptive Control and Signal Processing, 21, 2007. To appear.

[15] T. Söderström, M. Hong, and W. X. Zheng. Convergence properties of biaseliminating algorithms for errors-in-variables identification. International Journal of Adaptive Control and Signal Processing, 19(9):703-722, November 2005 .

[16] T. Söderström and P. Stoica. System Identification. Prentice Hall International, Hemel Hempstead, UK, 1989. 
[17] S. Van Huffel, editor. Recent Advances in Total Least Squares Techniques and Errors-in-Variables Modelling. SIAM, Philadelphia, USA, 1997.

[18] S. Van Huffel and Ph. Lemmerling, editors. Total Least Squares and Errorsin-Variables Modelling. Analysis, Algorithms and Applications. Kluwer, Dordrecht, The Netherlands, 2002.

[19] W. X. Zheng. Transfer function estimation form noisy input and output data. International Journal of Adaptive Control and Signal Processing, 12:365380, 1998. 